\title{
Consequences of Neural Asynchrony: A Case of Auditory Neuropathy
}

\author{
N. Kraus ${ }^{1}$, A.R. Bradlow ${ }^{2}$, M.A. Cheatham ${ }^{3}$, J. Gunningham ${ }^{3}$, C.D. $\mathrm{KinG}^{3}$, D.B. KoCH ${ }^{3}$, \\ T.G. NiCOL ${ }^{3}$, T.J. MCGEE ${ }^{3}$, L.K. STEIN ${ }^{3}$, AND B.A. WRIGHT ${ }^{3}$ \\ ${ }^{1}$ Departments of Communication Sciences; Neurobiology and Physiology; Otolaryngology, Northwestern University, \\ Evanston, IL 60208, USA \\ ${ }^{2}$ Department of Linguistics, Northwestern University, Evanston, IL 60208, USA \\ ${ }^{3}$ Department of Communication Sciences, Northwestern University, Evanston, IL 60208, USA
}

Received: 4 January 2000; Accepted: 9 March 2000; Online publication: 31 May 2000

\begin{abstract}
The neural representation of sensory events depends upon neural synchrony. Auditory neuropathy, a disorder of stimulus-timing-related neural synchrony, provides a model for studying the role of synchrony in auditory perception. This article presents electrophysiological and behavioral data from a rare case of auditory neuropathy in a woman with normal hearing thresholds, making it possible to separate audibility from neuropathy. The experimental results, which encompass a wide range of auditory perceptual abilities and neurophysiologic responses to sound, provide new information linking neural synchrony with auditory perception. Findings illustrate that optimal eighth nerve and auditory brainstem synchrony do not appear to be essential for understanding speech in quiet listening situations. However, synchrony is critical for understanding speech in the presence of noise.

Keywords: auditory neuropathy, asynchrony, auditory, perception, neural synchrony
\end{abstract}

\section{INTRODUCTION}

Auditory pathway dysfunction can provide insights into the neuroanatomical and physiological processes

Correspondence to: Dr. N. Kraus $\bullet$ Northwestern University $\bullet$ Frances Searle Building • 2299 N. Campus Dr. • Evanston, IL 60208. Telephone: 847 491-3165; fax: 847 491-2325; e-mail: nkraus@nwu.edu that underlie normal hearing. Auditory neuropathy is a hearing disorder in which peripheral hearing appears normal, but the eighth nerve and brainstem are abnormal (Davis and Hirsh 1979; Kraus et al. 1984; Starr et al. 1991). By clinical definition, patients with this disorder have normal otoacoustic emissions (OAEs) and cochlear microphonic (CM) potentials, but exhibit an absent or severely abnormal auditory brainstem response (ABR) (Starr et al. 1996). Because a normal $\mathrm{ABR}$ is recorded only when multiple neurons fire synchronously at stimulus onset, patients with auditory neuropathy provide an opportunity to examine the role of synchrony in perception.

The electrophysiological tests for diagnosing auditory neuropathy can be used in very young children, allowing this disorder to be identified early in life. The functional ramifications remain unclear, with reports ranging from functional deafness to relatively intact speech perception in quiet but severely impaired perception in noise (Davis and Hirsh 1979; Worthington and Peters 1980; Lenhardt 1981; Chisin et al. 1979; Kraus et al. 1984, 1993; Starr et al. 1991, 1996, 1998; Sininger et al. 1995; Berlin et al. 1993b, 1994, 1998; Berlin and Hood 1993a; Berlin 1996; Picton et al. 1981; Picton 1986; Stein et al. 1996). Because the preponderance of the reported cases are very young children, there is a paucity of information on the capabilities of adult auditory neuropathy patients. These descriptions are needed in order to (1) understand the effects of neural synchrony on perceptual abilities and central nervous system function, and (2) provide guidelines for treatment and intervention that are based on rea- 
sonable predictions of the hearing deficits that auditory neuropathy patients may experience.

An in-depth understanding of the specific perceptual deficits associated with neural asynchrony requires a comprehensive approach. One case of psychophysical abilities associated with auditory neuropathy was reported by Starr and colleagues (1991). Temporal processing deficits were evident. Gap detection was poor (threshold of $100 \mathrm{~ms}$ versus the normal $2 \mathrm{~ms}$ ) and was much worse for short- than for longer-duration stimuli. Binaural signal processing was impaired as indicated by elevated thresholds for the discrimination of interaural time and intensity differences, and the absence of a binaural masking level difference with the dichotic inversion of phase. Frequency and intensity discrimination of pure tones was also impaired, with the subject requiring 3-15 times the normal frequency change and twice the normal intensity change to discriminate a difference. Despite these deficits, the subject demonstrated relatively normal discrimination of stimulus duration. Unfortunately, Starr and colleagues did not address how these impaired psychoacoustic abilities related to speech perception.

This article describes the auditory capabilities of an adult with auditory neuropathy who has normal hearing sensitivity. Because understanding the perceptual consequences of auditory neuropathy is usually complicated by elevated auditory thresholds, this young woman provides a rare opportunity to examine speech perception, as well as other auditory abilities, in the absence of peripheral hearing loss. Her performance also offers guidance into which measures may yield the most insight into the perceptual deficits or strengths that influence speech perception in natural communication settings. A combined behavioralneurophysiological, acoustic-phonetic approach is taken to investigate the biological processes involved in the perception of speech sounds (Kraus et al. 1996, 1998, 1999; Koch et al. 1999a; Carrell et al. 1999; Bradlow et al. 1999). This experimental approach, coupled with psychophysical measures involving simple tones and noise bursts, reveals new information about the role of neural synchrony in auditory perception and the perceptual profile that may accompany a neural synchrony disorder.

This investigation is important because neural synchrony is a fundamental neurobiologic process underlying sensory, motor and cognitive events. Although internally generated synchronization of neural discharges has been linked to sensory/motor/cognitive processing and to attentional states that may be independent or loosely coupled to external stimulus events (Riehle et al. 1997; Stopfer et al. 1997; Barinaga et al. 1998; Llinas et al. 1988), it is the neural synchrony, directly elicited by external stimulation, that is considered here. Synchrony related to stimulus timing is

\begin{tabular}{l} 
TABLE 1 \\
\hline \multicolumn{1}{c}{ Test battery. } \\
\hline Audiometric tests \\
Pure-tone thresholds \\
Tympanometry and acoustic reflexes \\
Otoacoustic emissions \\
Brainstem-evoked audiometry \\
Behavioral perception in quiet \\
Sentence comprehension \\
Fine-grained speech-sound perception \\
Behavioral perception in degraded listening conditions \\
Word identification \\
Detection of tones in noise \\
Speech-elicited cortical potentials \\
Mismatch negativity response \\
P1/N1/P2 cortical responses \\
\hline
\end{tabular}

especially important in the auditory system (Eggermont 1990; Phillips 1993; Sinex et al. 1991), where the spectral and temporal complexity of a signal such as speech elicits responses from a broad neural population resulting in patterns of synchronized activity. Eggermont $(1990,1991,1997)$ has stressed the importance of neural synchrony across populations of neurons in the signaling of differences between steadystate and dynamic stimuli. In addition, synchronized aggregate neural responses have been shown to reflect perceptually important acoustic features in speech (McGee et al. 1996; Steinschneider et al. 1994).

\section{METHODS AND RESULTS}

The skills evaluated range from the perception of speech at the sentence and word levels to the perception of elemental speech sounds and tones. Measures of perception were made in quiet and in noise. Where appropriate, findings were viewed in the context of the sound structure of the signals, and the physiological activity evoked along the auditory pathway. Because of the large number of tests performed (Table I), the methods and results of each measure were combined.

\section{Case history}

IT is a 24-year-old woman who speaks both English and Hebrew. Her medical history is largely unremarkable. A neuropsychological evaluation revealed normal intellectual and academic performance. She earned a B.A. in psychology, followed by 2 years in an Outward Bound program. She plans to study public health in graduate school. She is a bright, cooperative adult who has good insight into her condition. IT's consent to participate in this study was obtained according to the declaration of Helsinki and with the 
approval of Northwestern University's Institutional Review Board.

IT's parents suspected that something was wrong with their daughter's hearing early in childhood. She was regarded as a "spaced out" child who often ignored obvious acoustic events. For example, she would not realize that the radio was playing only static. She was, however, sensitive to loud sounds, frequently requesting that the volume be lowered. Throughout her childhood, hearing tests indicated normal sensitivity, so her auditory symptoms were dismissed.

As an adult, IT reports that she is deaf in noisy environments. She describes herself as being cut off from sound by an imaginary wall. She cannot carry on a conversation in a car and has been frustrated by attempts to play an instrument or to sing, although she enjoys music. During college, she sat in the front row and had a note-taker because she could not hear the professor if she had to look down to take notes. She found it difficult to learn in large classes, and eventually transferred to a small college where class size was limited. She notices no change in hearing with fever, hot weather, or menstrual cycle. IT's father (and his mother before that) also complained of hearing problems, specifically severe difficulty hearing in noisy environments.

We previously evaluated IT when she was 18 years old (Kraus et al. 1993). That report included conventional audiometric results, electrophysiological recordings, an auditory processing test, and discrimination of selected phonemic contrasts. Magnetic resonance imaging revealed diffuse enlargement of the ventricular system but was otherwise normal. At that time, we were able to tell her that her auditory problems were not imagined and that, based on her ABR, her auditory system was not functioning normally. This information was of considerable comfort to her.

Audiogram, tympanogram, acoustic reflexes, and otoacoustic emissions

Conventional audiometry demonstrated bilaterally symmetric, normal pure-tone thresholds $(15,10,5$, and $5 \mathrm{~dB} \mathrm{HL}$ at $0.5,1,2$, and $4 \mathrm{kHz}$, respectively), normal speech-reception thresholds, and normal speech discrimination in quiet. Tympanometry revealed normal middle-ear function. Acoustic reflexes were absent, possibly contributing to her hyperacusis. Distortion product otoacoustic emissions (DPOAE) were obtained using the ILO92 system. DPOAEs were present in both ears for F2 frequencies between 1.5 to $6.0 \mathrm{kHz}$ (Fig. 1, bottom). Emissions exceeded the noise floor, indicated by the shaded region, by an average of $20 \mathrm{~dB}$.

\section{Auditory brainstem response}

Methods. Stimuli were clicks and 10-ms tone bursts of 1,2 , and $4 \mathrm{kHz}$ (2 cycles rise/fall time), presented monaurally at $70 \mathrm{~dB}$ nHL at a rate of $31.1 / \mathrm{s}$. Responses were recorded separately to stimuli at starting phases of $0^{\circ}$ and $180^{\circ}$. Neural activity was recorded from each ear (Cz-ipsilateral earlobe, forehead ground). Each response (minimum of $4 /$ stimulus condition) reflected an average of 2000 stimulus presentations.

Results. Responses for each ear were bilaterally symmetric. As shown in Figure 1 (top), click-evoked responses revealed an early oscillatory component that reversed phase when the stimulus was inverted. However, response latency did not shift with stimulus intensity or rate, as would be expected if the waves reflected neural responses. With tone-burst stimulation (not shown), the response showed oscillations matched to the stimulus frequency. These response properties are characteristic of hair cell, not neural potentials, and are thus thought to reflect the cochlear microphonic (CM) (Dallos 1973). In other words, the waveforms are dominated by presynaptic responses with the result that no ABR waves are identified.

The ABR of IT's father is also abnormal, although not as impaired as IT's. Despite a normal audiogram, the father's ABR thresholds were at $35 \mathrm{~dB}$ HL. Wave I was absent. Although wave III was observed inconsistently, when present, waves III and V displayed poor morphology and were significantly delayed (0.6-0.8 $\mathrm{ms})$.

\section{Behavioral perception in quiet}

Speech perception in quiet appeared normal during informal conversation. IT scored $100 \%$ on the City University of New York sentence test (Boothroyd et al. 1985 ) in a quiet auditory-only condition. Perception in quiet was impaired, however, when fine-grained discrimination of synthetic speech stimuli, differing along a single acoustic-phonetic dimension, was assessed.

Fine-grained speech-sound discrimination. Methods: A Parameter Estimation by Sequential Tracking (PEST) paradigm (Taylor and Creelman 1967) was used to evaluate just noticeable differences (JNDs) for three synthesized consonant-vowel continua-/ba/ to /wa/, /da/ to /ga/, and /da/ to /ga/-with amplitude enhancement of the formant transitions [see Kraus et al. (1999) and Carrell et al. (1999) for detailed descriptions of stimuli, experimental procedures and normative data]. These continua were created using the Klatt synthesizer (Klatt 1980) and represent changes in either formant transition duration $(/ \mathrm{ba} /$ to /wa/) or differences in the third-formant onset frequency $(/ \mathrm{da} /$ to $/ \mathrm{ga} /)$. A four-interval forcedchoice procedure was used to prevent response bias. 
Response at $1.5 \mathrm{msec}$ is cochlear microphonic.

a. polarity of response flips with polarity of stimulus

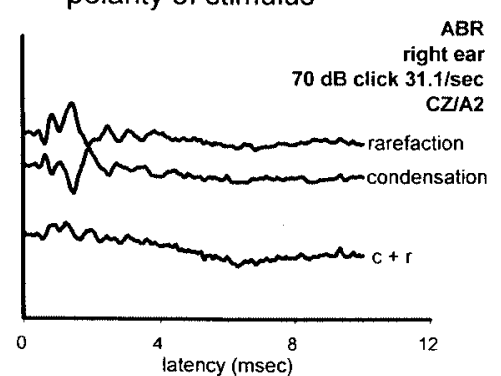

b. latency does not shift with stimulus intensity

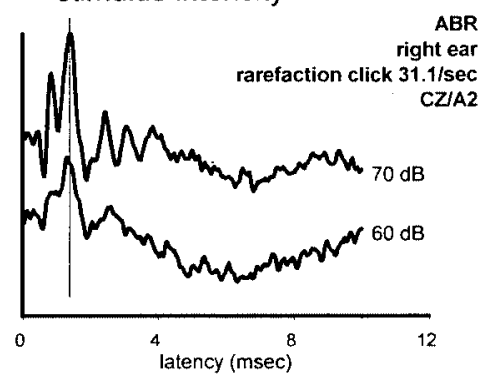

c. latency does not shift with stimulus rate

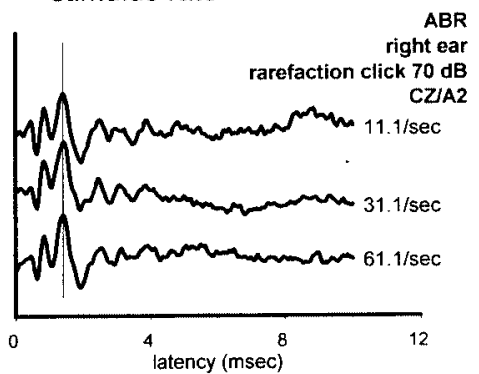

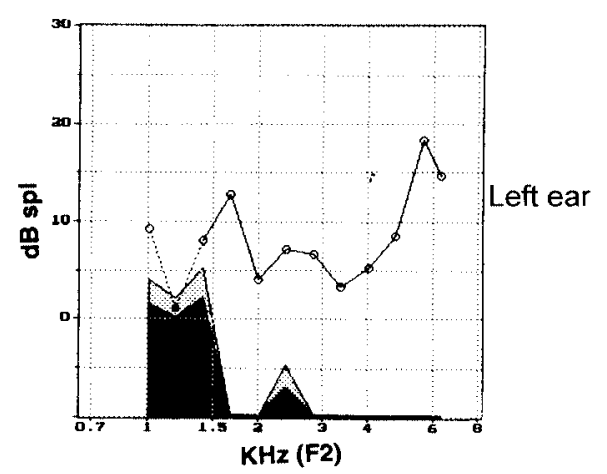

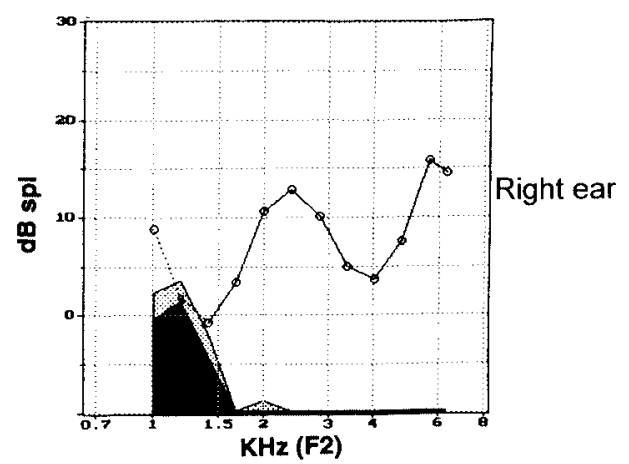

FIG. 1. (Top) The three panels illustrate the absence of ABR waves. The waveforms show only the cochlear microphonic because the polarity of response flips with the polarity of the stimulus (a), latency does not shift with stimulus intensity (b), and latency does not shift with stimulus rate (c). (Bottom) Although only right ear responses are shown, responses were bilaterally symmetric. OAE responses are present in each ear.

In each trial, IT was presented binaurally with two pairs of syllables where one pair was the same and one pair was different. The task was to indicate whether members of the first or the second pair of syllables were different. The order of same and different pairs within trials was randomized. A block ended when IT reached an accuracy level of $69 \%$ correct. Three trial blocks were obtained for each stimulus condition.

Results: For the /ba-wa/ continuum, IT had a remarkably good JND compared with normal adult listeners (Fig. 2). On average, normal adults discriminated stimuli in which the formant transition durations differed by at least $6 \mathrm{~ms}$. IT was able to discriminate stimuli in which the formant transition durations differ by just $3 \mathrm{~ms}$. In contrast, her JND for the /da-ga/ continuum was worse than that of normal adult listeners. Whereas normal adults discriminated stimuli in which the third formant onset frequencies differed by approximately $80 \mathrm{~Hz}$, IT required a difference of almost $120 \mathrm{~Hz}$. Moreover, when the /da-ga/ formant transition was enhanced in amplitude relative to the vowel, IT showed even greater difficulty discriminating along this continuum. In contrast, normal listeners had even better JNDs in the enhanced condition. Overall, the data suggest that IT is well able to discriminate synthetic speech stimuli that differ in the temporal domain (duration), but has difficulty discriminating stimuli that differ spectrally at stimulus onset and are characertized by rapid spectro-temporal changes throughout the formant transition. This pattern of results is similar to the pattern observed in some children with learning problems (see Discussion).

\section{Behavioral perception in degraded listening conditions}

Fine-grained speech-sound perception in noise. Methods and Results: Discrimination along another synthesized /da-ga/ continuum (stimuli were identical to /da-ga/ described above but contained onset burst frication during the first $10 \mathrm{~ms}$ at formants 3, 4, and 5 ) was tested in continuous Gaussian white noise (SNR $+10 \mathrm{~dB}$ ). IT was unable to discriminate these stimuli at all. Mean JNDs from 10 normal subjects are $140 \mathrm{~Hz}$, SDs 6.

Word identification. Methods: Monosyllabic word recognition was assessed binaurally across signal-to-noise ratios (SNR), across word lists spoken by either a single talker or multiple talkers, and across words that differ in inherent recognition difficulty. By manipulating these three factors-SNR, lexical difficulty, and single vs. multiple talkers-it is possible to investigate the effects on speech perception of multiple sources of 


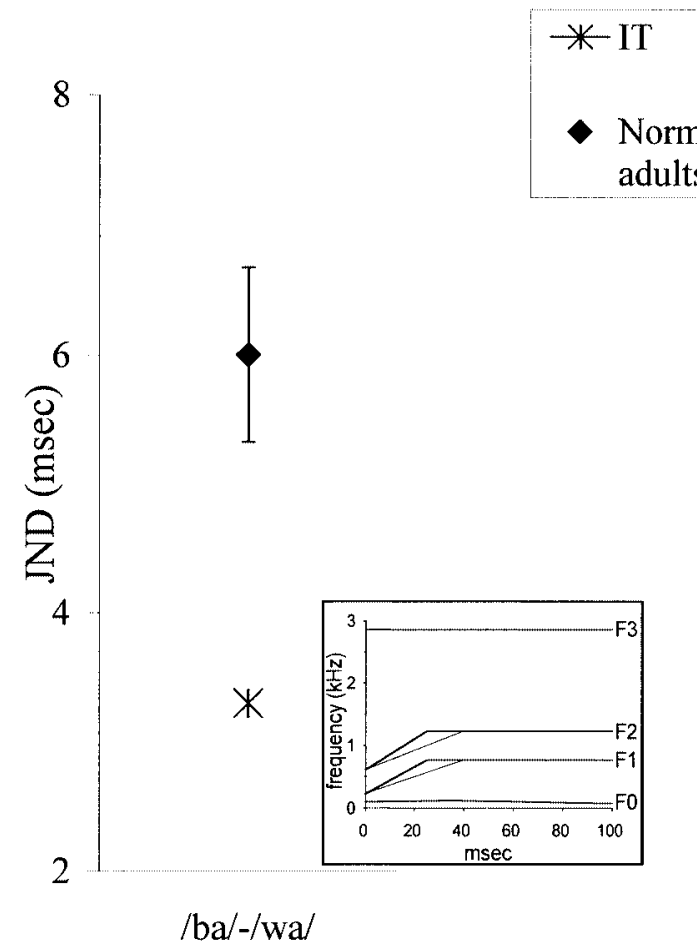

FIG. 2. Just noticeable differences for two synthetic speech continua for normal young adults ( $n=12$, means and standard errors) and IT. Normal adult means and SDs are $6 \mathrm{~ms}(2), 77 \mathrm{~Hz}(35)$ and $55 \mathrm{~Hz}$ (19) for/ba-wa/,/da-ga/ and/da-ga/-enhanced stimuli, respectively; see also Kraus et al. 1999). The /ba-wa/ continuum varied in the duration of the formant transition. The /da-ga/ continuum varied in

variability and signal degradation. These factors introduce precisely the signal variability and degradation encountered in real-world speech perception.

Using a word database developed at Indiana University (Torretta 1996; Bradlow and Pisoni 1999), two lists of 72 words each were created. IT heard each word through a loudspeaker (at $65 \mathrm{~dB}$ SPL) in a soundtreated room and then repeated the word, which was written down by the experimenter. Within each list, SNR was progressively decreased across four 18 -word sublists from $+12 \mathrm{~dB}$ to $+9 \mathrm{~dB}$ to $+6 \mathrm{~dB}$, and finally to $+3 \mathrm{~dB}$. Words in the first list were spoken by a single female talker, while words in the second list were spoken by nine different talkers ( 5 males, 4 females). Each list was composed of 36 "easy" words and 36 "hard" words. The words were categorized as easy or hard based on lexical characteristics identified by the Neighborhood Activation Model (NAM) of spoken word recognition (Luce 1986; Luce and Pisoni 1998). According to this model, an easy word is a word that has few similar-sounding "neighbors" with which it can be confused. Furthermore, these few lexical neighbors have a lower frequency of occurrence in the language than the word itself. In contrast, a hard word has many, high-frequency similar-sounding lexical neighbors with which it can be confused. Using a variety of spoken
160

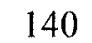

120

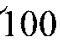

80

60

40

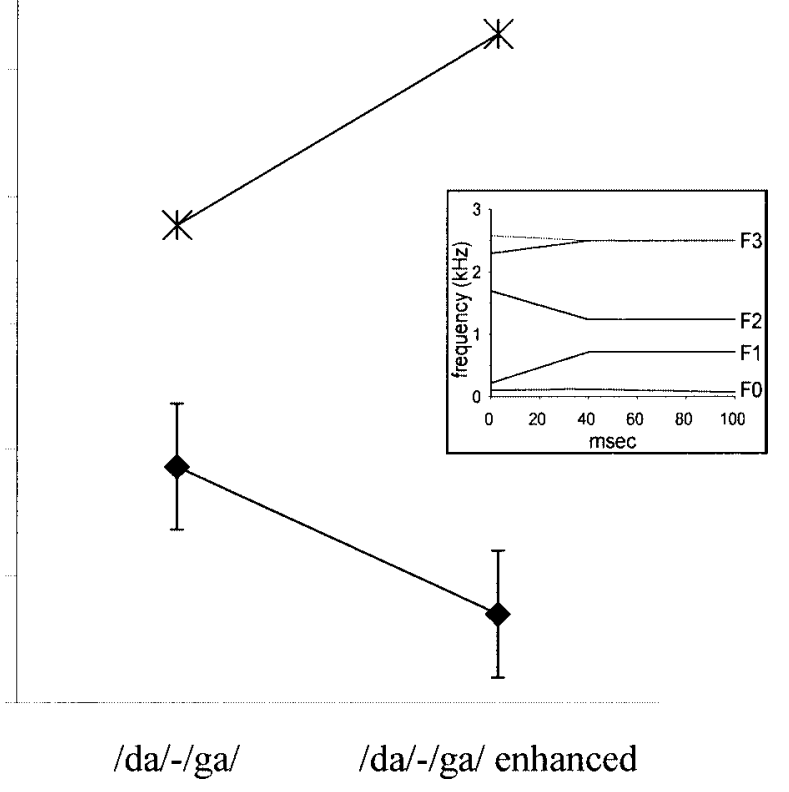

the onset frequency of the third formant with a 40-msec formant transition duration. The /da-ga/-enhanced continuum was the same as the /da-ga/ continuum but the amplitude of the formant transition was increased. The inserts show schematic spectrograms of key elements of the prototype phonemes. Thin lines represent/da/ and /wa/ and thick lines represent /ga/ and /ba/, respectively.

word recognition tasks, this easy-hard difference has been shown to have dramatic effects on word intelligibility (Pisoni et al. 1985; Luce 1986; Luce et al. 1990; Luce and Pisoni 1998). The intelligibility of the lists were equated, based on word recognition data from a large number of normal hearing subjects collected as part of the database development (Bradlow and Pisoni 1999).

Signal-to-noise ratio Results: Word identification scores across the four SNRs $(+12,+9,+6,+3 \mathrm{~dB})$ from a group of 15 normal subjects using the same lists and testing procedures as used for IT (from Koch et al. 1999b) are shown in Figure 3, along with the scores for IT. These scores are averaged across singleand multiple-talker presentation formats, and across easy and hard words. The pattern of decreasing scores with increasing noise was similar for IT and normal adults, although IT showed marked effects of noise at a larger SNR than normals. At the most favorable SNR $(+12 \mathrm{~dB})$, IT's performance was similar to that of normals. However, at all other, less favorable SNRs, IT's performance was substantially lower than the normal adults. Most notably, at $+3 \mathrm{~dB}$ SNR, IT was severely impaired, with only $10 \%$ correct word identification in comparison to the normal subjects who scored $\sim 40 \%$ correct. 


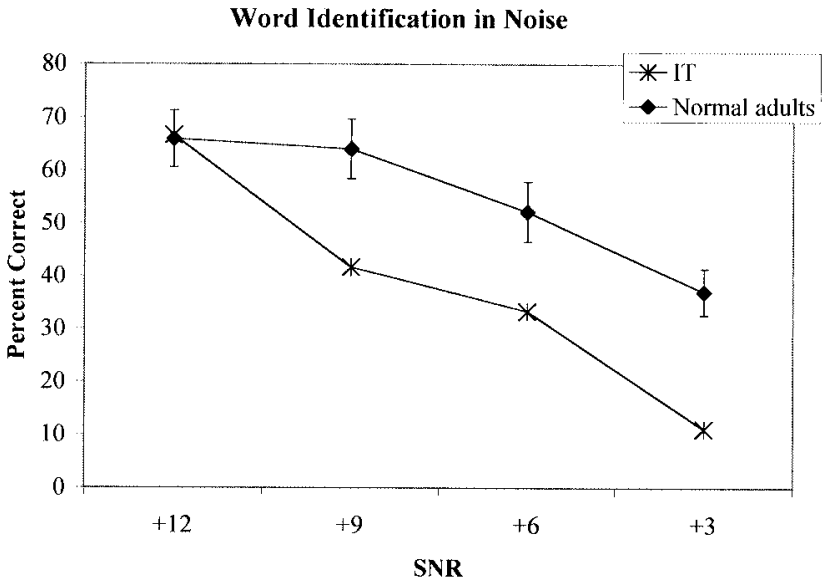

FIG. 3. Percent correct word identification scores are shown for four signal-to-noise ratios (SNR). Means and standard errors are shown for a group of 12 young adults. IT's scores are indicated by asterisks. IT's scores fall within the average at lower SNRs. Her performance is worse than the adult average at decreasing SNRs. Normal adult means and 1 standard deviation are 66 (19), 64 (19), 52(20), and 37 (15) for $+12,+9,+6$, and $+3 \mathrm{~dB}$ SNR, respectively.

Talker variability/lexical difficulty Results: Data, averaged across all four SNRs, are summarized in Figure 4. Like normals, IT was able to make use of the signal consistency provided by a single talker compared with the changing talker information in the multiple-talker condition. This suggests that, even in noise, she is sensitive to the nonlinguistic, qualitative aspects of the signal and is able to adapt to a speaking style to the extent that it can assist speech perception ability. The single-talker/multiple-talker effect was most noticeable for the "hard" words, suggesting that IT follows the normal pattern of perceiving words in the context of other words in the English lexicon. Like normal adults, the situation that presents the most difficulty is when the target word has many similar-sounding "competitors" and the surface signal characteristics (talker) change from item to item.

Detection of tones in noise. Methods: Following Wright et al. (1997), threshold was determined for a 20-ms tone of $1000 \mathrm{~Hz}$, presented either before or during a masking noise. Two noise types were employed: a bandpass noise that ranged from 600 to $1400 \mathrm{~Hz}$, and a spectrally notched noise that ranged from 400 to $800 \mathrm{~Hz}$ and from 1200 to $1600 \mathrm{~Hz}$. Both noises were $300 \mathrm{~ms}$ in duration and had a spectrum level of $40 \mathrm{~dB}$ SPL. All stated durations include 10-ms cosine-squared gating envelopes.

The tone and noise were digitally generated in the frequency domain using a digital-signal-processing board (TDT AP2; sampling rate $25 \mathrm{kHz}$ ). They were delivered separately through two 16-bit digital-to-analog converters (TDT DD1) followed by separate 8.5kHz low-pass filters (TDT FLT5), separate programmable attenuators (TDT PA4), and a single sound mixer

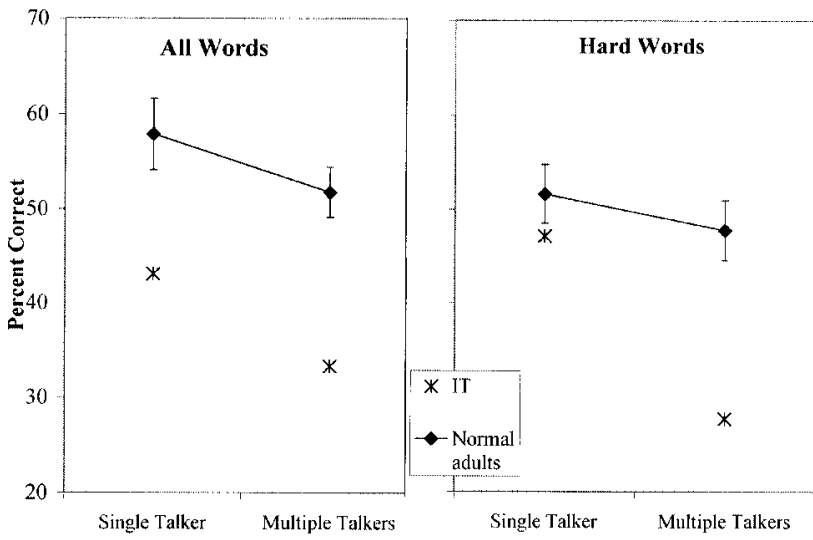

FIG. 4. The effect of single vs. multiple talkers on word identification. Means and standard errors for a group of normal adults are indicated by the filled symbols. IT's word identification scores were consistently better in the single-talker compared with the multipletalker condition. Her performance was severely affected when acoustic complexity was further increased in the "hard" word condition. IT's scores are indicated by asterisks. Normal mean percent correct scores and standard deviations are 58 (4) and 52 (3) for All Words and 52 (3), 48 (3) for Hard Words, single and multiple talkers, respectively.

(TDT SM3) and headphone driver (TDT HB6). The listener was seated in a sound-treated room and listened monaurally through the left earpiece of Sennheiser HD450 headphones.

Thresholds were measured in three conditions. In the backward-masking condition, the tone ended immediately before the beginning of the bandpass noise. Performance in that condition provides an estimate of how well the listener can separate sounds in time. In two simultaneous-masking conditions, the tone was presented $200 \mathrm{~ms}$ after the beginning of either the bandpass or spectrally notched noise. The threshold difference between those two conditions provides an estimate of how well the listener can separate sounds in frequency (Patterson et al. 1982).

The method was two-interval forced-choice with feedback. Observation intervals were separated by 800 ms. Signal level was adjusted adaptively using a maximum-likelihood method, which estimated the tone level required for $94 \%$ correct detections after 30 trials (Green 1990). Two threshold estimates were obtained in each condition, but one estimate in the simultaneous-masking condition with the bandpass noise was omitted due to technical problems. Neither IT nor the comparison listeners had any previous experience with the conditions.

Results: Backward masking. IT's threshold in the backward-masking condition was $51 \mathrm{~dB}$ SPL. That value is considerably higher than the mean of about $32 \mathrm{~dB}$ SPL (SDs 6, range 23-41 dB SPL) for 10 adults with normal hearing tested in the same condition (Hartley et al., in review). Thus, IT is poorer than normal at separating sounds in time.

Results: Simultaneous masking. IT's thresholds in the 
simultaneous-masking conditions were $79 \mathrm{~dB}$ SPL with the bandpass noise and $75 \mathrm{~dB}$ SPL with the spectrally notched noise. This compares to means of about 72 dB SPL (SDs 4, range 65-77 dB SPL) and $54 \mathrm{~dB}$ SPL (SDs 7, range 47-70 dB SPL), respectively, for 10 adults with normal hearing tested in the same conditions (Hartley et al., in review). The threshold difference between the bandpass and spectrally notched noise was only $3.5 \mathrm{~dB}$ for IT, but was about $18 \mathrm{~dB}$ for adults with normal hearing. Thus, IT is much poorer than normal at separating a brief tone from noise components that are remote from the tone in frequency. IT's overall higher thresholds may indicate that she is a less efficient listener than normal adults (Patterson et al. 1982), which is consistent with IT's difficulty hearing in noisy environments.

\section{Speech-elicited cortical potentials}

$\mathrm{P} 1 / \mathrm{N} 1 / \mathrm{P} 2$ responses to stimuli differing in voice onset time (VOT). Method: The stimuli were two synthetic syllables, /ba/ and / pa/, each with a duration of 320 ms. The syllables were composed of five formants. The steady-state values of F1-F5 were 720, 1240, 2500, 3600, and $4500 \mathrm{~Hz}$. For $/ \mathrm{ba} /$, there was no burst and the transition duration was $40 \mathrm{~ms}$. The starting frequencies of F1 and F2 were 220 and $900 \mathrm{~Hz}$. For /pa/ a 10-ms burst followed by aspiration began at $5 \mathrm{~ms}$, remained constant until $40 \mathrm{~ms}$, and decreased to zero at $45 \mathrm{~ms}$. Full-amplitude voicing began at $40 \mathrm{~ms}$.

To elicit $\mathrm{P} 1 / \mathrm{N} 1 / \mathrm{P} 2$ responses, each syllable was presented 250 times with an interstimulus interval of $1090 \mathrm{~ms}$. Responses were recorded using scalp electrodes placed at $\mathrm{Fz}$ (active), on the nose (reference), and on the forehead (ground). For each stimulus, responses were averaged across the 250 presentations.

Results: As shown in Figure 5, IT had robust P1/ $\mathrm{N} 1 / \mathrm{P} 2$ potentials and her response to / ba/ was similar to that observed in normal subjects. Consistent with the normal pattern, latencies for IT differed for $/ \mathrm{ba} /$ and /pa/. However, $\mathrm{P} 1$ and $\mathrm{N} 1$ responses to / pa/ occurred later than the normal mean for IT. In normal subjects, cortical responses to voiceless stimuli have two peaks, one related to the aspiration/burst (labeled P1' in the figure), the second to the onset of voicing (Sharma and Dorman 1999; Koch et al. 1997). This early peak was absent in IT. Thus, while timing differences were apparent between IT's responses to $/ \mathrm{ba} /$ and $/ \mathrm{pa} /$, irregularities in the representation of these signals also occurred. Perceptually, IT's finegrained discrimination of stimuli along a /ba-pa/ continuum was similar to that observed for normal subjects (JND $=1.2$; normal adult mean $=2$, SDs 1.6; $n=10)$. Unfortunately, information about her perception of these stimuli in noise is not available.
Mismatch response to speech contrasts differing in formant spectrum and duration. Methods: The mismatch negativity (MMN) has been purported to be a measure of processes associated with auditory discrimination, because it is the change in acoustic stimulation that triggers the occurrence of the response [Näätänen et al. 1978; see Näätänen (1992) and Kraus et al. (1995a) for reviews]. It is also thought to reflect processes associated with echoic memory (Näätänen et al. 1989). Two speech contrasts used in the behavioral finegrained speech-sound discrimination experiments/ba-wa/ and / da-ga/_-were presented in an oddball paradigm to the right ear at $75 \mathrm{~dB}$ SPL through insert earphones as previously described (Kraus et al. 1996). For each MMN, 3500 stimuli were presented with a rare-stimulus probability of 10-15\%. Responses also were obtained to a sequence in which the rare stimulus was presented repetitively for 2000 trials (rare alone). The MMN was calculated as the difference between the responses to a stimulus presented as a rare stimulus in the oddball paradigm and that same stimulus presented alone in a repetitive sequence. Thus, a difference wave is obtained which is not confounded by inherent stimulus differences.

Results: Robust mismatch responses of normal morphology, duration, area, scalp distribution and hemispheric symmetry were obtained to /ba-wa/ contrasts, consistent with IT's excellent behavioral perception of stimuli along a / ba/ to /wa/ continuum. MMN was absent in response to the /da-ga/ contrast (Fig. 6). Thus, processes reflecting discrimination and echoic memory of acoustic stimuli were intact when elicited by stimuli differing in formant duration but not by stimuli differing in formant onset frequency. These responses are consistent with her overall poor perception of sounds along the /da/-to-/ga/ continuum.

\section{DISCUSSION}

\section{Auditory nerve and brainstem synchrony in perception}

This case illustrates the extent to which speech perception can be preserved and how it is impaired when auditory pathway synchrony is compromised. In quiet, IT's speech perception was excellent at the sentence and word levels, where contextual and multiple acoustic cues are available. Her speech perception in quiet was abnormal only when the discrimination of finegrained, stripped-down phonetic elements was required. In noise, IT's speech perception was markedly impaired. She demonstrated poor performance on a variety of speech perception measures including the perception of syllables, words with increasing levels of 


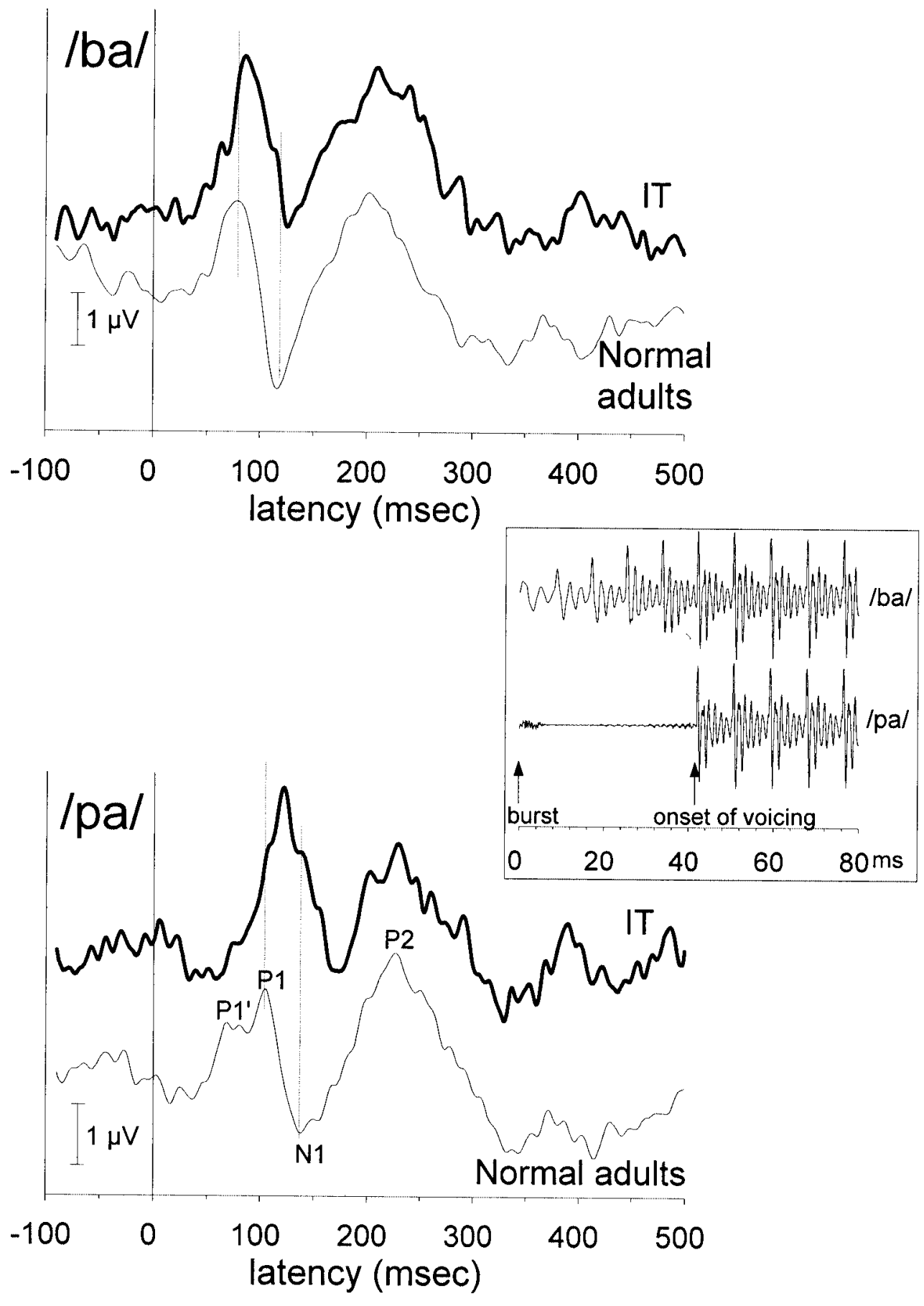

FIG. 5. P1/N1 cortical responses to stimuli differing in voice onset time (/ba/ and /pa/). In each panel, the top thick line is IT's response. The bottom thin line is the grand average response to each stimulus from 11 normal adults. The dashed vertical lines reflect normal mean $\mathrm{P} 1$ and $\mathrm{N} 1$ response latencies. IT's responses to $/ \mathrm{ba} /$ are similar to the normal mean, while $\mathrm{P} 1$ and $\mathrm{N} 1$ responses to $/ \mathrm{pa} /$ occurred later (121 and $174 \mathrm{~ms}$, respectively) than normal mean latencies. In addition, wave P1' was absent for IT. Normal adult means and SDs for $/ \mathrm{pa} /$ are 99 (16) and 146 (17) ms for P1 and N1 latencies, respectively. The insert shows the salient acoustic differences between the /ba/ and /pa/ stimuli. background noise, multiple talkers, and hard words where phonetic confusions are most likely.

IT's difficulties with fine-grained phonetic distinctions and with speech perception in noise are consistent with her problems separating simple sounds in both time and frequency. The psychophysical data suggest a CNS contribution to frequency coding. The abnormal detection thresholds obtained in the simultaneous masking condition demonstrate a deficit in the ability to separate a tone from noise components that are remote from the tone in frequency. This deficit is unlikely due to abnormal cochlear mechanics (i.e., degraded frequency selectivity of peripheral origin) since otoacoustic emissions were shown to be present in both ears.

This case provides a link between stimulus-timingrelated neural synchrony and perception. It illustrates that excellent speech perception is possible in quiet with an absent ABR. Optimal eighth nerve and auditory brainstem synchrony do not appear to be essential 


\section{/ba-wal}
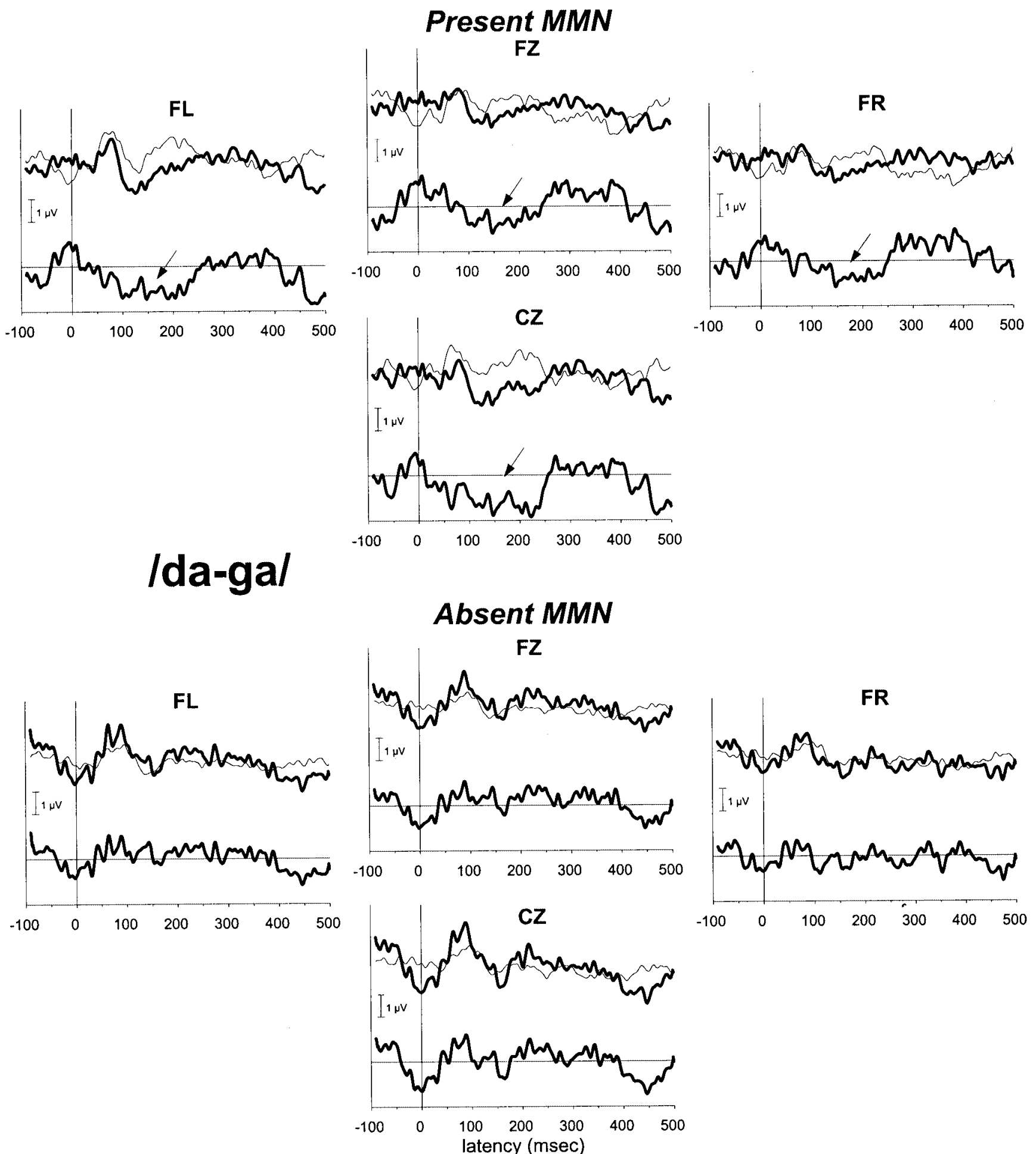

FIG. 6. Grand average $M M N$ responses elicited by a /ba/-/wa/ and $\mathrm{a} / \mathrm{da} /-/ \mathrm{ga} /$ contrast from central ( $\mathrm{Fz}$ and $\mathrm{Cz}$ ) and lateral electrode locations ( $\mathrm{FI}$ and $\mathrm{Fr}$ ). In the four panels at the top of the figure, the top thin line is the response to a $/ \mathrm{wa} /$ stimulus when it was presented alone. The top thick line is the response to a /wa/ stimulus when it signaled an acoustic change in a sequence of /ba/ stimuli. In the four panels at the bottom of the figure, the top thin line is the response

to $\mathrm{a} / \mathrm{da} / \mathrm{stimulus}$ when it was presented alone. The top thick line is the response to a /da/ stimulus when it signaled an acoustic change in a sequence of /ga/ stimuli. In both columns, the $M M N$ is seen in the difference wave (lower thick line) as a deflection below the zero line. Normative data for all MMN parameters (duration, area, amplitude, onset latency) in response to these stimuli are found in Kraus et al. (1999). 
for understanding speech in ideal listening situations. Results also indicate that synchrony is critical for understanding speech in the presence of noise, which is what we do most of the time. Nevertheless, it must be noted that the absence of stimulus-timing-related synchrony in IT does not preclude the presence of other forms of synchrony in neural assemblies which exist without precise time-locking to external stimuli or in relation to internally generated cognitive events (Riehle et al. 1997; Barinaga et al. 1998; Stopfer et al. 1997).

\section{Subcortical synchrony and cortical function}

Germane to the consideration of neural synchrony is the assumption that evoked responses reflect synchronous activity across a population of neurons. It should be emphasized, however, that cortical potentials reflect neural synchrony differently than the ABR. The ABR peaks reflect synchronous spike discharges generated in nerve tracts, whereas the peaks in cortical responses reflect the summation of excitatory postsynaptic potentials. In other words, the ABR reflects action currents in axons, while the cortical potentials reflect slow dendritic events. These differences are evident in the spectra of these evoked potentials: the dominant peak in the ABR is $\sim 1 \mathrm{kHz}$ (Boston and Moller, 1985), whereas the peak for cortical potentials is on the order of tens of hertz (Moller 1994). Because unit contributions to the ABR are biphasic and of short duration, ABR peaks tend to cancel when discharges are separated by fractions of a millisecond. In contrast, for cortical potentials, the waves are so slow that contributions separated by several milliseconds contribute to these later waves. While the ABR reflects highly synchronous discharges with microsecond precision, the synchrony required for cortical potentials is on the order of several milliseconds. These differences demand that the rate of stimulation be slower for cortical than for brainstem responses (e.g., 2/s vs. 30/s, respectively), limiting the precision with which timing information can be represented. Nevertheless, a more rapid rate of stimulus presentation alone cannot account for the absent ABR in IT and in other individuals with auditory neuropathy (Starr et al. 1996). The $\mathrm{ABR}$ is absent even when the stimulation rate is as slow as for cortical responses.

For IT, auditory function central to the brainstem, as reflected by cortical-evoked responses, was largely preserved despite abnormal input from lower centers. That is, middle latency responses, $\mathrm{P} 1 / \mathrm{N} 1 / \mathrm{P} 2, \mathrm{MMN}$, and P300 cortical responses were robust and present in quiet (Kraus et al. 1993). Cortical potentials deviated from normal, however, when elicited by finegrained, temporal aspects of speech-sound structure. For example (as shown in Fig. 6), IT had absent MMN to changes in formant onset frequency (/da-ga/). Perhaps the neural representation of these signals depends on the precise neural synchrony known to characterize single neuron activity in auditory cortex in response to the onset of acoustic signals (Phillips 1993; Wang et al. 1995). It is interesting to consider that a similar impairment in perception and neural representation of the spectro-temporal formant changes exemplified by /da-ga/ is seen in children with learning problems (Kraus et al. 1996). Although it has been postulated that impairment of the temporal processing by cortical mechanisms underlies deficits in the ability to discriminate rapid spectro-temporal transitions (Tallal 1981; Nagarajan et al. 1999, Kraus et al. 1996), recent findings also point to a subcortical origin (e.g., brainstem) for these deficits (Cunningham et al. 2000). A high degree of temporal precision is also required to encode rapid formant transitions (Phillips et al. 1989; Phillips and Hall 1990). In the case of IT, subcortical input may not have been sufficiently synchronous to be utilized effectively at more central levels for the perception of the more difficult acoustic discriminations.

Cortical representation of voice onset time (VOT) has been demonstrated in single and aggregate intracranial neuron activity (Eggermont 1995, Steinschneider et al. 1994, McGee et al. 1996). In humans, voiceless stimuli elicit two peaks, one reflecting the initial stimulus burst, and a later one reflecting the onset of voicing (Sharma and Dorman 1999; Koch et al. 1997). Asynchrony of subcortical input may have contributed to IT's atypical cortical representation of VOT as shown in Figure 5. The absence of the early peak $\left(\mathrm{P} 1^{\prime}\right)$ to / pa/ suggests poor representation of transient onset cues which may be related to the stimulus burst. Nevertheless, adequate representation of longer-duration voicing cues, related to harmonic stimulus features, may have contributed to the relative timing differences between her /ba/ and / pa/ responses and her good behavioral perception of stimuli along a VOT continuum.

Finally, a fundamental property of the central nervous system is its inherent plasticity. It is apparent that the central pathways can make use of varied and limited input that can be interpreted as speech by the brain. For example, with deprivation or altered peripheral sensory input, cortical areas which would ordinarily represent input from a peripheral sensory system continue to be active, even though that peripheral system is impaired. In fact, cortical representation reorganizes, and the representation of intact aspects of sensory input is expanded (Irvine and Rajan 1996; Merzenich et al. 1991; Kitzes 1984; Reale et al. 1987; Popelar et al. 1994). Perhaps IT is able to "fill in" missing information based on cortical reorganization resulting from atypical input from auditory brainstem 
pathways. It should be noted that IT's cortical potentials were particularly well-formed in amplitude and morphology. Moreover, her perception of fine-grained differences along a / ba-wa/ continuum was extraordinarily good, possibly reflecting neural reorganization and developed compensatory abilities. Future studies, which address cortical reorganization associated with auditory neuropathy, may shed some light on these speculations.

\section{Representation of timing information in speech}

Rosen (1992) and Phillips (Phillips et al. 1989; Phillips and Farmer 1990) have provided frameworks for considering speech signals in the time domain. For example, suprasegmental components of speech typically are expressed over hundreds or thousands of milliseconds, periodicity of the fundamental frequency occurs with roughly a $10-\mathrm{ms}$ period, and the fine-structure of speech, characteristic of many consonants, occurs in the tenths-of-millisecond range. This case supports the notion that the synchrony disorder primarily affects the representation of precise timing information occurring at stimulus onset. ABR by definition is a response to stimulus onset and reflects neural information in the tenth-of-millisecond range. In fact, the timing information is so robust that latency delays on the order of $0.2 \mathrm{~ms}$ are considered abnormal clinically. However, in addition to affecting responses to stimulus onset, impairment also may extend to other forms of stimulus-timing-related neural synchrony such as the representation of offset responses and phase locking to harmonic and steady-state aspects of the stimulus.

It should be noted that IT had perceptual difficulty in response to sounds containing critical acoustic information at stimulus onset, rather than to stimuli requiring discrimination of durational cues within a syllable. Specifically, discrimination thresholds for synthetic CV syllables revealed that IT had exceptionally good discrimination for speech sounds along a /ba-wa/ continuum but poor discrimination for /da-ga/ contrasts. The difference between / da/ and /ga/ occurs at stimulus onset while the difference between /ba/ and /wa/ occurs within the syllable. Cortical responses to these stimuli revealed a similar pattern of strengths and weakness (to / ba-wa/ and /da-ga/, respectively) in the neural representation of these stimuli. IT's atypical cortical representation of /pa/ further supports the existence of deficits in the representation of transient stimulus cues related to the onset of voiceless syllables, whereas longer-duration harmonic aspects of voicing appeared to be preserved (perhaps via phase locking). Furthermore (as shown in Fig. 2), normal-hearing adults seem to be helped by increasing the amplitude of the formant transition relative to the vowel (enhanced transition), whereas
IT was not. Auditory neurons in cortex increase synchrony and precision of firing with increases in stimulus intensity (Phillips and Hall 1990). Possibly IT cannot profit from this mechanism because, in her case, neural input into auditory cortex lacks onset precision in the first place.

This subject, however, was able to extract speechsound cues in quiet. She exhibited normal sentence and word identification performance when the listening conditions were favorable (a SNR of at least $+12 \mathrm{~dB}$ ). Moreover, her relatively better perception of words in a single-talker condition, relative to a multiple-talker condition, suggests that she is able to make efficient use of the talker-specific consistencies in the acoustic signal (e.g., voice/source, individual articulatory characteristics that remain relatively stable across items in a list, fundamental frequency) to help her extract important linguistic information.

Overall, the representation of timing information at stimulus onset appeared most vulnerable to disruption, with representation of longer-duration and steady-state timing cues being better preserved. The neural representation and perception of all signals tested, however, was fragile and easily disrupted by noise. It appears that over a wide range of stimuli, IT cannot make use of neural mechanisms (e.g., 8th nerve) that ordinarily represent the temporal structure of the speech signal (Kiang and Moxon 1974; Delgutte and Kiang 1984a, b, c), and that have been shown to be particularly important when speech is presented in noise.

\section{CONCLUSION}

IT's profile may provide a "best-case scenario" of how well a person with auditory neuropathy can perform. Although her perceptual deficits and strengths should not be construed as a characterization of all neuropathy patients, they do raise interesting questions and provide clues from which to speculate about the role of neural synchrony in perception. Clearly, a normal audiogram does not indicate normal hearing and an absent ABR does not necessarily indicate elevated behavioral thresholds. Given the alterations in neural activity between the ear and the brain that occur in this case of auditory neuropathy, it is remarkable that hearing and speech perception in quiet are still possible.

The test results reported here imply that the auditory cortex can adjust to the faulty signal representations present at earlier stages along the auditory pathway. In fact, the grossly intact cortical potentials recorded in this case suggest that patients with auditory neuropathy can use varied and limited inputs to perceive complex signals. Further application of the 
comprehensive experimental approach used here may help to elucidate the perceptual consequences associated with disorders of neural synchrony, as well as the neurophysiological mechanisms underlying normal and impaired auditory function.

\section{ACKNOWLEDGMENTS}

This work was supported by NIH-NIDCD DC01510. The authors thank IT and her family for their time and valuable insight.

\section{REFERENCES}

BARINAGA M. Listening in on the brain. Science. 280:376-378, 1998. BERLIN CI, HOOD LJ. Pseudo-central hearing loss: a confusion of central for peripheral hearing loss caused by faulty conditioning techniques and lax criteria. Sem. Hear. 14:215-223, 1993a.

Berlin CI, Hood LJ, Cecola RP, Jackson D, Szabo P. Afferentefferent disconnection in humans. Hear. Res. 65:40-50, 1993b.

Berlin CI, Hood LJ, Hurley A, Wen H. Contralateral suppression of otoacoustic emissions: an index of the function of the medial olivocochlear system. Otolaryngol. Head Neck Surg. 110:3-21, 1994.

BERLIN CI. Role of infant hearing screening in health care. Sem. Hear. 17:115-124, 1996.

Berlin C, Bordelon J, St John P, Wilensky M, Hurley A, Kluka E, Hood L. Reversing click polarity may uncover auditory neuropathy in infants. Ear Hear. 19:37-47, 1998.

Boothroyd A, Hanin L, Hnath T. A sentence test of speech perception: reliability, set equivalence, and short term learning. Internal report RCI 10, 1985; Speech and Hearing Sciences Research Center, City University of New York.

Boston J, Moller A. Brainstem auditory-evoked potentials. CRC Crit. Rev. Biomed. Eng. 13:97-123, 1985.

Bradlow A, Kraus N, Nicol T, McGee T, Cunningham J, Carrell T. Effects of lengthened transition duration on discrimination and neural representation of synthetic CV syllables by normal and learning-disabled children. J. Acoust. Soc. Am. 106:20862096, 1999.

Bradlow A, Pisoni D. Recognition of spoken words by native and non-native talkers: Talker-, listener- and item-related factors. J. Acoust. Soc. Am. 106:2074-2085, 1999.

CARRELl TD, BRADLOW AR, Nicol T, Koch DB, KRAUS N. Interactive software for evaluating auditory discrimination. Ear. Hear. 20:175176, 1999.

Chisin R, Perlman M, Sohmer H. Cochlear and brain stem hearing loss following neonatal hyperbilirubinemia. Ann. Otol. Rhinol. Laryngol. 88:352-357, 1979.

CunNINGHAM J, NiCOL T, KRAUS N. Neurophysiologic representation of clear speech in noise. Assoc. Res. Otolaryngol. 2000. [Abstract].

Dallos P. The Auditory Periphery. Academic Press, New York, NY, 1973.

DAVIS H, HiRsh S. A slow brainstem response for low-frequency audiometry. Audiology. 18:445-461, 1979.

DelgutTe B, KIANG NYS. Speech coding in the auditory nerve: I. Vowel-like sounds. J. Acoust. Soc. Am. 75:866-878, 1984a.

DeLgutTe B, KIANG NYS. Speech coding in the auditory nerve: III. Voiceless fricative consonants. J. Acoust. Soc. Am. 75:887-896, 1984b.

DelgutTe B, KIANG NYS. Speech coding in the auditory nerve: V.
Vowels in background noise. J. Acoust. Soc. Am. 75:908-918, 1984c.

Eggermont J. The Correlative Brain: Theory and Experiment in Neural Interaction. Studies of Brain function. Vol 16. SpringerVerlag, Berlin, 1990.

EGGERMONT J. Rate and synchronization measures of periodicity coding in cat primary auditory cortex. Hear. Res. 56:153-167, 1991.

EGGERMONT J. Representation of a voice onset time continuum in primary auditory cortex of the cat. J. Acoust. Soc. Am. 98:911920,1995

EGGERMONT J. Firing rate and firing synchrony distinguish dynamic from steady state sound. Neuroreport. 8:2709-2713, 1997.

GREEN DM. Stimulus selection in adaptive psychophysical procedures. J. Acoust. Soc. Am. 87:2662-2674, 1990.

Hartley DEH, Hogan SC, Wright BA, Moore DR. Age related improvements in auditory backward and simultaneous masking in 6-10 year old children (submitted).

IRVINE DR, RAJAN R. Injury- and use-related plasticity in the primary sensory cortex of adult mammals: possible relationship to perceptual learning. Clin. Exp. Pharmacol. Physiol. 23:10-11, 939947, 1996.

KIANG NY, Moxon EC. Tails of tuning curves of auditory-nerve fibers. J. Acoust. Soc. Am. 55:620-630, 1974.

King C, Nicol T, McGee T, Kraus N. Thalamic asymmetry is related to acoustic signal complexity. Neurosci. Lett. 28:89-92, 1999.

Kitzes L. Some physiological consequences of neonatal cochlear destruction in the inferior colliculus of the gerbil, Meriones unguiculatus. Brain Res. 306:171-178, 1984.

KLATT DH. Software for a cascade/parallel formant synthesizer. J. Acoust. Soc. Am. 67:971-995, 1980.

Koch D, Tremblay K, Dunn I, Dinces E, Carrell T, Kraus N. Speech-evoked N1 and mismatch neurophysiologic responses in cochlear-implant users and normal listeners. Assoc. Res. Otolaryngol. Abstr. 20:80, 1997.

Koch DB, McGee TJ, Bradlow AR, Kraus N. Acoustic-phonetic approach toward understanding neural processes and speech perception. J. Am. Acad. Audiol. 10:304-318, 1999a.

Koch DB, Bradlow AR, Bellis TJ, Kraus N. Effects of age on speech-sound representation and perception in normal-hearing women. Assoc. Res. Otolaryngol. Abstr. 22:73, 1999b.

Kraus N, Özdamar Ö, Stein L, Reed N. Absent auditory brain stem response: Peripheral hearing loss or brain stem dysfunction? Laryngoscope. 94:400-406, 1984.

Kraus N, McGee T, Ferre J, Hoeppner J, Carrell T, Sharma A, NICOL T. Mismatch negativity in the neurophysiologic/behavioral evaluation of auditory processing deficits: a case study. Ear. Hear. 14:223-234, 1993

Kraus N, McGee T, Littman T, Nicol T, King C. Nonprimary auditory thalamic representation of acoustic change. J. Neurophysiol. 72:1270-1277, 1994.

Kraus N, McGee T, Carrell T, Sharma A. Neurophysiologic basis of speech discrimination. Ear. Hear. 16:19-37, 1995a.

Kraus N, McGee T, Carrell T, King C, Tremblay K, Nicol T. Central auditory system plasticity associated with speech discrimination training. J. Cogn. Neurosci. 7:25-32, 1995b.

Kraus N, McGee TJ, Carrell TD, Zecker SG, Nicol TG, Koch DB. Auditory neurophysiologic responses and discrimination deficits in children with learning problems. Science. 273:971-973, 1996.

Kraus N, McGeE T, Koch D. Speech-sound representation, perception and plasticity: A neurophysiologic perspective. Audiol. Neurootol. 3:168-182, 1998.

Kraus N, Koch D, McGee T, Nicol T, Cunningham J. Speechsound discrimination in school-age children: Psychophysical and neurophysiologic measures. J. Speech. Hear. Lang. Res. 42:1042_ 1060, 1999. 
LENHARDT M. Childhood central auditory processing disorder with brainstem evoked response verification. Arch. Otolaryngol. 107:623-625, 1981.

LLINAS R. The intrinsic electrophysiological properties of mammalian neurons: insights into central nervous system function. Science. 242:1654-1664, 1988.

LuCE P. (1986) Neighborhoods of words in the mental lexicon. Research on Speech Perception, Technical Report No. 6. Indiana University, Bloomington, IN.

LUCE P, PIsONI D. Recognizing spoken words: the neighborhood activation model. Ear. Hear. 9:1 1-36, 1998.

LUCE P, PISONI D, GOLDINGER S. Similarity neighborhoods of spoken words. Altmann G, Cognitive models of speech processing: psycholinguistics and computational perspectives. MIT Press, Cambridge, MA, 1990, 122-147.

McGee T, Kraus N, King C, NiCOL T. Acoustic elements of speechlike stimuli are reflected in surface recorded responses over the guinea pig temporal lobe. J. Acoust. Soc. Am. 99:3606-3614, 1996.

Merzenich M, Grajski K, Jenkins W, Recanzone G, Peterson B. Functional cortical plasticity: cortical network origins of representational changes. Cold Spring Harbor Symp. Quant. Biol. 55:873887, 1991.

MOLLER A. Neural generators of auditory evoked potentials. JACOBsON JT. Principles and Applications in Auditory Evoked Potentials Allyn and Bacon Boston 1994, 23-46.

NÄÄTÄNEN R. Attention and Brain Function Erlbaum Association, Hillsdale, NJ, 1992, 136-200.

NÄÄTÄNEN R, GAILlaRd AW, MaNTYSALO S. Early selective-attention effect on evoked potential reinterpreted. Acta. Psychol. (Amst.). 42:313-329, 1978.

NÄÄTÄNEN R, PAAVILAINEN P, REINIKAINEN K. Do event-related potentials to infrequent decrements in duration of auditory stimuli demonstrate a memory trace in man? Neurosci. Lett. 107:347352, 1989.

Nagarajan S, Mahncke H, Salz T, Tallal P, Roberts T, Merzenich M. Cortical auditory signal processing in poor readers. Proc. Natl. Acad. Sci. 96:6483-6488, 1999.

Patterson RD, Nimmo-smith I, Weber DL, Milroy R. The deterioration of hearing with age: Frequency selectivity, the critical ratio, the audiogram, and speech threshold. J. Acoust. Soc. Am. 72. 1788-1803, 1982.

PHILLIPS DP. Representation of acoustic events in the primary auditory cortex. J. Exp. Psychol. Hum. Percept. Perform. 19:203216, 1993.

PhilliPs DP, FARMer ME. Acquired word deafness, and the temporal grain of sound representation in the primary auditory cortex. Behav. Brain. Res. 15:85-94, 1990.

Phillips D, Hall S. Response timing constraints on the cortical representation of sound time structure. J. Acoust. Soc. Am. 88:1403-1411, 1990.

Phillips DP, Hall SE, Hollett JL. Repetition rate and signal level effects on neuronal responses to brief tone pulses in cat auditory cortex. J. Acoust. Soc. Am. 85:2537-2549, 1989.

Picton T. Abnormal brainstem auditory evoked potentials: A tentative classification. In Evoked Potentials AR Liss, Inc. New York, NY, 1986, 373-378.

Picton TW, StaPells DR, CAmpbell KB. Auditory evoked potentials from the human cochlea and brainstem. J. Otolaryngol. Suppl. 9:1-41, 1981.
Pisoni D, Nusbaum H, Luce P, Slowiaczek L. Speech perception, word recognition and the structure of the lexicon. Speech Communication 4:75-95, 1985.

Popelar J, Erre J, Aran J-M, Cazals Y. Plastic changes in ipsicontralateral differences of auditory cortex and inferior colliculus evoked potentials after injury to one ear in the adult guinea pig. Hear. Res. 72:125-134, 1994.

ReAle R, BRugge J, Chan C. Maps of auditory cortex in cats reared after unilateral cochlear ablation in the neonatal period. Brain Res. 431:281-290, 1987.

Riehle A, Grun S, Diesmann M, Aertsen A. Spike synchronization and rate modulation are differentially involved in motor cortical function. Science. 278:1950-1953, 1997.

Rosen S. Temporal information in speech: acoustic, auditory and linguistic aspects. Philos. Trans. R. Soc. Lond. B. Biol. Sci. 336:367-373, 1992.

SHARMA A, Dorman M. Cortical auditory evoked potential correlates of categorical perception of voice-onset-time. J. Acoust. Soc. Am. 106:1078-1083, 1999.

Sinex D, McDonald L, MotT J. Neural correlates of nonmonotonic temporal acuity for voice-onset-time. J. Acoust. Soc. Am. 90:24412449, 1991.

Sininger YS, Hood LJ, Starr A, Berlin CI, Picton TW. Auditory loss due to auditory neuropathy. Audiol. Today. 7:10-13, 1995.

Starr A, McPherson D, Patterson J, Luxford W, Shannon R, SININGER Y, TONOKAWA L, WARING M. Absence of both auditory evoked potentials and auditory percepts dependent on time cues. Brain. 114:1157-1180, 1991.

Starr A, Picton TW, Sininger Y, Hood LJ, Berlin CI. Auditory Neuropathy. Brain. 119:741-753, 1996.

Starr A, Sininger Y, Winter M, Derebery MJ, Oba S, Michalewski HJ. Transient deafness due to temperature-sensitive auditory neuropathy. Ear Hear. 19:169-179, 1998.

Stein L, Tremblay K, Pasternak J, Banerjee S, Lindemann K, Kraus N. Brainstem abnormalities in neonates with normal otoacoustic emissions. Sem. Hear. 17:197-213, 1996.

Steinschneider M, Arezzo JC, Schroeder C, Vaughan HG. Jrw Speech evoked activity in primary auditory cortex: effects of voice onset time. Electroenceph. Clin. Neurophysiol. 92:30-43, 1994.

Stopfer M, Bhagavan S, Smith B, Laurent G. Impaired odour discrimination of desynchronization of odour-encoding neural assemblies. Nature. 390:70-74, 1997.

TALLAL P. Language disabilities in children: perceptual correlates. Int. J. Pediatr. Otorhinolaryngol. 3:1-3, 1981.

Taylor M, CreElman C. PEST: Efficient estimates on probability functions. J. Acoust. Soc. Am. 41:782-787, 1967.

ToRetTA G. The "Easy-Hard" word multi-talker speech database: an initial report. Res. Spoken. Lang. Process. Prog. Rep. 20:321334, 1996.

Wang X, Merzenich MM, Beitel R, Schreiner CE. Representation of a species-specific vocalization in the primary auditory cortex of the common marmoset: temporal and spectral characteristics. J. Neurophysiol. 74:2685-2706, 1995.

Worthington D, Peters J. Quantifiable hearing and no ABR: Paradox or error? Ear Hear. 5:281-285, 1980.

Wright BA, Lombardino LJ, King WM, Puranik CS, Leonard CM, MERZENICH MM. Deficits in auditory temporal and spectral resolution in language-impaired children. Nature 387:176-178, 1997. 